\title{
Acknowledgment to Reviewers of Antioxidants in 2020
}

\section{Antioxidants Editorial Office}

Citation: Antioxidants Editorial

Office. Acknowledgment to

Reviewers of Antioxidants in 2020.

Antioxidants 2021, 10, 170. https://

doi.org/10.3390/antiox10020170

Published: 25 January 2021

Publisher's Note: MDPI stays neutral with regard to jurisdictional claims in published maps and institutional affiliations.

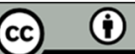

Copyright: (C) 2021 by the author. Licensee MDPI, Basel, Switzerland. This article is an open access article distributed under the terms and conditions of the Creative Commons Attribution (CC BY) license (http://creativecommons.org/licenses /by/4.0/).

MDPI AG, St. Alban-Anlage 66, 4052 Basel, Switzerland

Peer review is the driving force of journal development, and reviewers are gatekeepers who ensure that Antioxidants maintains its standards for the high quality of its published papers. Thanks to the cooperation of our reviewers, in 2020, the median time to first decision was 14 days and the median time to publication was 33 days. The editors would like to express their sincere gratitude to the following reviewers for their precious time and dedication, regardless of whether the papers were finally published:

Abdelmohsen, Usama Ramadan

Abenavoli, Ludovico

Abraham, Nader

Abu-Reidah, Ibrahim M.

Acedo, Jeella Z.

Ačkar, Đurđica

Acquah, Caleb

Acuña-Castroviejo, Darío

Adamakis, Ioannis-Dimosthenis

Adamek, Agnieszka

Adamska-Patruno, Edyta

Adigun, Oludoyin

Adornetto, Annagrazia

Afonso, Andrea Luísa Fernandes

Agnetti, Giulio

Agnieszka, Szopa

Aichinger, Georg

Aiello, Francesca

Aimo, Alberto

Aires, Alfredo

Aitken, R. John

Alaimo, Alessandro

Alamprese, Cristina

Albano, Emanuele

Albertini, Maria

Albrecht, Jan

Alcaraz, María José

Aldasoro, Martin

Alexovič, Michal

Allegra, Mario

Allerton, Timothy

Alloatti, Giuseppe

Alloza, Iraide

Allu, Prasanna K.R.

Al-Maharik, Nawaf
Almajano, María Pilar

Al-Mrabeh, Ahmad

Almstrup, Kristian

Alonso-Alconada, Daniel

Alonso-Moreno, Carlos

Alonso-Torre, Sara

Alquezar-Burillo, Carolina

Altamimi, M.

Alvarez Fernandez, María Antonia

Alvarez-Suarez, Jose

Alves, C. Henrique

Alves-Lopes, Rheure

Alves-Silva, Jorge M

Amaral, Alexandra

Amaral, Joana S.

Amarowicz, Ryszard

Amillis, Sotiris

Amiya, Eisuke

Ammendola, Rosario

Amoaku, Winfried M.K.

Amodio, Nicola

Amorati, Riccardo

Amorós, Asunción

Ampofo, Emmanuel

Anandhan, Annadurai

Ancuceanu, Robert

Andrade, Isabel

Andre, Filipa

Andrei, Sanda

Andreou, Eleni

Angelino, Donato

Angeloni, Giulia

Angius, Fabrizio

Anifandis, George

Anisimova, Irina 
Annibalini, Giosuè

Annunziata, Giuseppe

Ansari, Rais

Antal, Diana

Antipova, Veronica

Antognelli, Cinzia

Antolak, Hubert

Anton, Pauline

Antonelli Incalzi, Raffaele

Antonini, Giovanni

Appell, Michael

Aprile, Francesco A.

Aprodu, Iuliana

Aratani, Yasuaki

Araujo, Maria E.M.

Arcone, Rosaria

Arellano, Juan B.

Arese, Marzia

Arguelles, Sandro

Arguello, Jose

Arion, Felix

Arita, Masanori

Armand, Anne-Sophie

Armand, Martine

Armeni, Tatiana

Armindo Melo, Armindo

Arndt, Patrick G.

Arner, Elias

Arnhold, Juergen

Arsene, Andreea Letiția

Artín-Aragón, Sagrario M.

Assari, Shervin

Aston, Kenneth I.

Asuero, Agustín G.

Atalay, Mustafa

Athanasiadis, Vassilis

Athanasiou, Labrini V.

Atlante, Anna

Audrito, Valentina

Augé, Nathalie

Aydillo, Carlos

Aykin-burns, Nukhet

Azab, Walid

Azadi, Hossein

Azzimato, Valerio

Azzini, Elena

Babickova, Janka

Bacchetti, Tiziana

Bacci, Stefano

Bach, Duc-Hiep

Bąchor, Remigiusz
Bácskay, Ildikó

Badea, Mihaela

Badino, Paola

Bae, Dong-Hun

Bae, Hae-Rahn

Bae, Jeehyeon

Bae, Ok-Nam

Bagci, Soyhan

Bagdziunas, Gintautas

Bagnicka, Emilia

Bagnoli, Paola

Bai, Renren

Bailey, Charles G

Bajek, Anna

Bajinskis, Ainars

Bajor, Malgorzata

Baki, Gabriella

Balan, Prabhu

Balasubramanian, Balamuralikrishnan

Balcerczyk, Aneta

Baldanzi, Gianluca

Baldisserotto, Anna

Bálint, Erika

Bališ, Peter

Balzano, Tiziano

Bambling, Matthew

Bandyopadhyay, Debasish

Banerjee, Aditi

Banti, Christina N.

Barba, Francisco J.

Barbosa-Pereira, Letricia

Barbouti, Alexandra

Barca, Amilcare

Bárcena, José Antonio

Barker, Tyler

Barragán, Montserrat

Barreca, Davide

Barreto, Maria Carmo

Barritt, Greg

Bartella, Lucia

Bartelt, Alexander

Bartolini, Susanna

Bartosz, Grzegorz

Bartoszewski, Rafal

Baschieri, Andrea

Basile, Adriana

Basile, Teodora

Baskaran, Rathinasamy

Basu, Reetobrata

Batchelor, Paul

Batinic-Haberle, Ines 
Battafarano, Giulia

Battistuzzi, Giannantonio

Bauer, Georg

Bauer, Inge

Bauer, Mario

Bax, Bridget E.

Baydur, Ahmet

Bazwinsky-Wutschke, Ivonne

Beberok, Artur

Becatti, Matteo

Bednar, Petr

Bedon, Frank

Beharry, Kay D.

Behringer, Erik

Beis, Dimitris

Bellezza, Ilaria

Bellier, Jean Pierre

Bellosta, Paola

Belyaeva, Olga V.

Bendich, Adrianne

Benedec, Daniela

Benedetti, Francesca

Benhar, Moran

Benito, José Manuel

Bennett, ALAN B.

Benohoud, Meryem

Benoist, Hervé

Benson, David

Bentel, Jacqueline M.

Berardesca, Enzo

Berardo, Clarissa

Bergantini, Laura

Berger, Daniela Cristina

Berger, Trish

Beriáin Apesteguía, María José

Bermudez, Marcel

Bernabò, Nicola

Bernardini, Giulia

Bernátová, Iveta

Bernocchi, Graziella

Bernstein, Nirit

Berreau, Lisa M.

Berti, Federico

Bertocchi, Martina

Bezirtzoglou, Eugenia

Bharath, Leena P.

Bhattacharyya, Piyali

Bhatti, Fazal-ur-Rehman

Biagini, Giuseppe

Białek, Agnieszka

Bialonska, Dobroslawa
Bianco, Armandodoriano

Bidault, Guillaume

Bielas, Wiesław

Bielle, Franck

Bijak, Michał

Bikov, András

Bilski, Jan

Bink, Diewertje I

Bird, Amanda

Birringer, Marc

Birsa, Mihail Lucian

Bisti, Silvia

Biswas, Arijit

Bito, Tomohiro

Bjerregaard, Poul

Bjornstedt, Mikael

Black, Stephen M.

Blackstone, Craig

Blais, Anne

Blalock, William

Blando, Federica

Blangetti, Marco

Blank, Ralf

Blanton, Cynthia

Blasi, Francesca

Blažević, Ivica

Bleilevens, Christian

Bloksgaard, Maria

Bloor, Stephen

Board, Mary

Boarescu, Paul-Mihai

Boaz, Mona

Bobis, Otilia

Bochkov, Valery

Bocian, Szymon

Boczkowska, Maja

Boffa, Luisa

Bogdański, Paweł

Böhm, Volker

Bohn, Torsten

Boltze, Johannes

Bombardi, Cristiano

Bömer, Nils

Boni, Raffaele

Bonini, Sara Anna

Bonior, Joanna

Bonnländer, Bernd

Bonsembiante, Federico

Boo, Yong Chool

Bora, Puran S.

Borbély, Yves 
Bordes, María Dolores Llobat

Bordin, Luciana

Bordonaro, Michael

Borghini, Andrea

Borkum, Jonathan M.

Borras, Consuelo

Borrelli, Emma

Bosca, Lisardo

Boschi-Muller, Sandrine

Botez, Elisabeta

Bottani, Emanuela

Bottone, Maria Grazia

Bouet, Valentine

Bourdon, Emmanuel

Bouropoulos, Nikolaos

Boyden, Penelope A.

Bozic, Josko

Bracco, Enrico

Bradshaw, Nicholas

Bradshaw, Patrick

Brady-Kalnay, Susann M.

Braidy, Nady

Braig, Simone

Brainina, Khiena Z.

Branco, Vasco

Brandli, Alice

Brandt, Kirsten

Brash, Alan

Brasili, Elisa

Bratek-Skicki, Anna

Bravo, Jeronimo

Bravo, Susana B.

Brčić Karačonji, Irena

Brebu, Mihai

Breitbart, Haim

Brestic, Marian

Brett, Thomas

Bromfield, Elizabeth

Broskey, Nicholas T.

Brown, Dennis

Brown, Kyle E.

Brown, LaVerne L.

Brownlee, Iain A.

Brukman, Nicolas

Brullo, Chiara

Brunetti, Cecilia

Brunetti, Giacomina

Brunetti, Luigi (Italy)

Brunetti, Luigi (USA)

Bruno, Laura

Bruns, Danielle R.
Brycki, Bogumil

$\mathrm{Bu}$, Pengli

Bucekova, Marcela

Bucevičius, Jonas

Buchet, Rene

Buchner, Peter

Buck, Leslie

Bucolo, Claudio

Budzisz, Elżbieta

Bueno Dalto, Danyel

Buentzel, Jens

Buey, Rubén M.

Buga, Ana

Buga, Ana-Maria

Bullon, Pedro

Bülow, Margret H.

Bunea, Andrea

Bunik, Victoria

Burcul, Franko

Burns, David

Burns, Teresa A.

Busco, Giovanni

Businaro, Rita

Butcher, Adrian

Butler, Thomas O.

Butterfield, David Allan

Caballero-Garcia, Beatriz

Cabeza, Laura

Cabiscol, Elisa

Caccamo, Daniela

Cacciola, Francesco

Cachofeiro, Victoria M.

Cádiz-Gurrea, María De La Luz

Cagliero, Cecilia Lucia

Cahill, Paul

Cai, Wenlong

Caiati, Carlo

Caiazzo, Elisabetta

Caires, Hugo R.

Cairo, Gaetano

Cairrão, Elisa

Calabrese, Edward J.

Calcabrini, Cinzia

Caldara, Marina

Caliceti, Cristiana

Calina, Daniela

Calvo-Lobo, César

Cametti, Cesare

Camins, Antoni

Cammisotto, Vittoria

Campbell, Fiona M. 
Campos Rosa, Joaquín María

Campos, Debora

Camps, Jordi

Cañadas, Olga

Cancello, Raffaella

Cangkrama, Michael

Canini, Antonella

Cannavò, Serafinella P.

Cantini, Francesca

Cao, Jian

Capasso, Raffaele

Capó, Xavier

Carbone, Katya

Cardenia, Vladimiro

Cardoso, Isabel Santos

Cardoso, Susana

Cardullo, Nunzio

Carelli, Stephana

Carles Escola-Gil, Joan

Carlisi, Daniela

Carlotto, Silvia

Carluccio, Maria Annunziata

Carmeli, Shmuel

Carmen, Mejía

Carnevale Neto, Fausto

Carnevale, Roberto

Caroppo, Ettore

Carotenuto, Marco

Carotti, Simone

Carradori, Simone

Carrera Fernández, Ceferino

Carrera, Mónica

Carresi, Cristina

Carrillo, Celia

Carrizzo, Albino

Carstensen, Hans Heinrich

Carullo, Gabriele

Caruso, Giulia

Carvalho, Andreia N.

Carvalho, Daniel

Carvelli, Lucia

Casalino, Elisabetta

Casamassimi, Amelia

Casanova, Llanos

Casanova-Sáez, Rubén

Casas, Rosa M.

Caseiro, Armando

Casella, Luigi

Caser, Matteo

Casey, Jennifer

Caspari, Thomas
Castellano, Immacolata

Castillo-Gonzalez, Claudia Marcela

Castñeiras, Alfonso

Catarino, Marcelo D.

Catucci, Gianluca

Caulfield, Thomas R.

Cauli, Omar

Cavaliere, Gina

Cavallaro, Sebastiano

Cavoski, Ivana

Cejkova, Jitka

Ceranowicz, Piotr

Cerqueira, Fatima

Cervantes, Emilio

Cervellati, Carlo

Cervelli, Manuela

Cervera, Ana

Cesa, Stefania

Cesaro, Annabelle

Chae, Heeyoung

Cháfer-Pericás, Consuelo

Chaki, Mounira

Chami, Belal

Chan, Catherine

Chand, Kirat K.

Chang, Cheng-Chung

Chang, Chia-che

Chang, Geng-Ruei

Chang, Hsin-I.

Chang, Hsueh-Wei

Chang, Jer-Ming

Chang, Kee-lung

Chang, Kun-Che

Chang, Ling-Chu

Chang, Te-Sheng

Chang, Yuan-Yen

Chano, Tokuhiro

Chao, Louis Kuoping

Chapple, Sarah

Chartoumpekis, Dionysios

Chatzinikolaou, Athanasios

Chatzopoulou, Paschalina

Chavatte, Laurent

Chekanov, Konstantin

Chen, Chiung-Mei

Chen, Chung-Hwan

Chen, Chun-Jung

Chen, David

Chen, Guibing

Chen, Hsiao-Jou Cortina

Chen, Hsing-Yin 
Chen, Jen-Tsung

Chen, Jing-Hsien

Chen, Liang-Yu

Chen, Shuen-Ei

Chen, Wei-June

Chen, Xiaoyan

Chen, Yihung

Chen, Yih-Yuan

Chénais, Benoit

Cheng, Juei-Tang

Cheng, Pei-Wen

Cheng, Yuan-Bin

Cheon, Yong-Pil

Cherkaoui Malki, Mustapha

Chhabra, Gagan

Chiang, Hsiu-Mei

Chiang, Wen-Dee

Chiaramello, Anne

Chimienti, Guglielmina Alessandra

Chinnici, Fabio

Chirikova, Nadezhda K.

Chistyakov, Vladimir Anatolievich

Chitgupi, Upendra

Chiu, Yi-Han

Chizzola, Remigius

Chmielarz, Piotr

Cho, Jae Youl

Cho, Jongki

Cho, Somi Kim

Chobot, Vladimír

Choi, Jonghoon

Choi, Jong-il

Choi, Yoon Kyung

Choi, Young Hae

Choi, Yun Sang

Chong, Cher-Rin

Chou, Wen-Hai

Chrzanowski, Grzegorz

Chua, Horng Ruey

Chung, Hun Taeg

Chung, Hwan-Suck

Chyau, Charng-Cherng

Ciaccio, Marcello

Cianciolo, Giuseppe

Ciarcia, Roberto

Cicchella, Antonio

Cicco, Paola De

Ciccodicola, Alfredo

Ciccone, Marco

Çiçek, Serhat Sezai

Cicero, Arrigo
Cichorek, Miroslawa

Cichowska, Joanna

Cicione, Claudia

Ciesla, Lukas

Cilla, Antonio

Cimino, Francesco

Cimmino, Giovanni

Cimpoiu, Claudia

Cioanca, Oana

Ciobica, Alin

Cione, Erika

Ciornea, Elena Todirascu

Čipak Gašparović, Ana

Cipak, Lubos

Cipolloni, Luigi

Ciz, Milan

Cladis, Dennis

Clanchy, Felix

Clemens, Mark

Clifford, Tom

Cocco, Raffaella

Cocco, Tiziana

Codella, Roberto

Codina, Georgiana

Cohen, Guy

Cole, Marsha

Colell, Anna

Colla, Emanuela

Collier, Jason

Colnaghi, Luca

Colonna, Maria Antonietta

Colucci, Giuseppe

Comi, Cristoforo

Comincini, Sergio

Comino, Penny

Comizzoli, Pierre

Constantinescu-Aruxandei, Diana

Constantinou, Violetta

Consumi, Marco

Contardi, Marco

Conte, Ivan

Contino, Marialessandra

Contreras, María Del Mar

Coombs, Melanie

Copeland, Paul

Copolovici, Dana Maria

Copolovici, Lucian

Coppo, Lucia

Coquerel, David

Corazzari, Marco

Cordani, Nicoletta 
Cordaro, Marika

Corpe, Christopher

Correia, Carlos M.

Corsi, Lorenzo

Corso, Giovanni

Cossignani, Lina

Costa, Raquel

Costa, Roberto

Costantini, Lara

Cotârleț, Mihaela

Cottrell, Jeremy

Couch, James R.

Coumans, Joëlle V. F.

Couture, Manon

Couturier, Jeremy

Cozar, I

Craig, Morrie

Craveiro, Rita

Cravero, Maria Carla

Cravotto, Giancarlo

Crespo, Irene

Crestani, Maurizio

Crinelli, Rita

Crispi, Stefania

Croft, Kevin D.

Cross, Neil

Crupi, Pasquale

Crupi, Rosalia

Cruz-Chamorro, Ivan

Csányi, Gábor

Cuchillo, Inmaculada

Cui, Ye

Cullen, Joseph

Cuomo, Francesca

Ćurko, Natka

Currò, Monica

Curti, Daniela

Curtis, Michael J.

Cutler, Mary Lou

D. Bortner, Carl

D'Amico, Stefano

D'Angelo, Livia

D'Angelo, Rosalia

D'Antona, Nicola

D'auria, Enza

D'Emmanuele Di Villabianca, Roberta

D'Orazi, Gabriella

D'Orazio, Giovanni

D'Orsi, Beatrice

Da Silva, Luís Pinto

Da Silva, Sabrina Daniela
Dacheux, Jean-Louis

Dagnell, Markus

Dal Monte, Massimo

Dalessandri, Domenico

Dalessandro, Giuseppe

Dall'Acqua, Stefano

Dall'Aglio, Cecilia

Dalla Pozza, Elisa

Damiano, Fabrizio

Dansette, Patrick M.

Dar, Wasim A.

Dario, Brunetti

Darmochwał-Kolarz, Dorota

Darvesh, Altaf

Daugelavicius, Rimantas

Dave, Sandeep

Davies, Michael

Davis, Keith R.

Davis, Randall L.

Dawra, Rajinder

Day, Andrew S.

De Bellis, Luigi

De Falco, Bruna

De Filippis, Barbara

De Geest, Bart

De Haan, Judy

De Leonardis, Antonella

De Lucca Camargo, Livia

De Martin, Sara

De Masi, Luigi

De Melo, Marcelo M.r.

De Tullio, Mario C.

De Vendittis, Emmanuele

De Waard, Vivian

De-Bandt, Jean-Pascal

Deckert, Joanna

Defant, Andrea

Degan, Paolo

Degl'innocenti, Donatella

Dekker, Marloes

Del Castillo-Santaella, Teresa

Delcourt, Nicolas

Delis, Costas

Dell' Agli, Mario

Delprat, Benjamin

Delvin, Edgard

De-Miguel, Manuel

Dent, Maja

Derry, Paul

Devesa, Jesús

Devkota, Hari Prasad 
Devkota, Pratima

DeWald, Tracy

Di Cerbo, Alessandro

Di Giacomo, Silvia

Di Gioia, Maria Luisa

Di Marco, Stefano

Di Maro, Antimo

Di Martino, Antonio

Di Mattia, Carla Daniela

Di Natale, Concetta

Di Nunzio, Mattia

Di Paola, Rosanna

Di Pietro, Natalia

Di Salle, Anna

Di Sotto, Antonella

Diaconeasa, Zorita

Diakopoulos, Nina

Diaz-Vivancos, Pedro

Dickerhof, Nina

Didion, Sean P.

Diederich, Sandra

Diego, La Mendola

Difonzo, Graziana

Dikalov, Sergey

Dimauro, Ivan

Dinh, Quynh Nhu

Dini, Irene

Dinica, Rodica-Mihaela

Dinis, Lia Tânia J

Dinis, Mário

Dintcheva, Nadka Tzankova

Direito, Rosa

DiSilvestro, Robert

Dobrikova, Anelia

Dodds, W. Jean

Dogossy, Gábor

Dolashka, Pavlina

Dołowy, Małgorzata

Domenicotti, Cinzia

Domínguez Medina, Eduardo

Dominguez, Juan Carlos

Domínguez-Álvarez, Enrique

Domínguez-Perles, Raúl

Domoki, Ferenc

Donati Zeppa, Sabrina

Donato, Luigi

Donato, Paola

Donato, Rosario

Donato, Rosario Francesco

Donnini, Sandra

Donno, Dario
Dos Santos, Jorge Luiz

Dosio, Franco

DOSOKY, NOURA

Drabińska, Natalia

Drenjančević, Ines

Drevinskas, Tomas

Drosten, Matthias

Drozd, Radoslaw

Drużyńska, Beata

Duarte, Amilcar

Duarte, Delfim

Dubrova, Yuri

Duda-Chodak, Aleksandra

Dudas, Jozsef

Dudley, Samuel C.

Duer, Stanisław

Dufossé, Laurent

Duggan, Brendan M

Dugue, Benoit

Duguez, Stephanie

Dulf, Francisc

Dumitru, Gabriela

Duncan, Raymond Scott

Duplessis, Mélissa

Durante, Miriana

Durante, William

Duranti, Guglielmo

Duszka, Kalina

Dutartre, Patrick

Dutu, Ligia

Duvigneau, J. Catharina

Dziadas, Mariusz Tomasz

Dziekońska, Anna

Dziki, Dariusz

Dżugan, Małgorzata

Eckhart, Leopold

Edge, Ruth

Efthimia, Cotou

Egawa, Tatsuro

Egerszegi, István

Eidenberger, Thomas

Eilertsen, Karl-Erik

El Ghoch, Marwan

El Midaoui, Adil

Elango, Jeevithan

Elgendy, Mohamed

Elisabetta, Bravi

Elshabrawy, Hatem

Elsner, Matthias

Emri, Eszter

Endre Károly, Kristóf 
Endres, Kristina

Engelberg, David

Engelhardt, Paul E.

Engevik, Melinda

Enns, Caroline

Erdélyi, Márta

Eri, Rajaraman

Esatbeyolgu, Tuba

Escames, Germaine

Escudero, Olga

Espadas Villanueva, Isabel

Espen, Luca

Espino, Javier

Espinoza, Jorge Luis

Esquifino, Ana Isabel

Esteve-Romero, Josep

Estrela, José M.

Eufemi, Margherita

Euler, Gerhild

Eva, Martínez-Pinilla

Evans, Clive

Evans, Kervin

Exbrayat, Jean-Marie

Ezhov, Marat

Fabiani, Roberto

Fadda, Angela

Faitot, Francois

Falcão, Soraia I.

Falnoga, Ingrid

Falqué López, Elena

Fang, Junnan

Fang, SungSoon

Fanizzi, Francesco Paolo

Fanti, Laura

Farabegoli, Fulvia

Farè, Silvia

Fato, Romana

Favati, Fabio

Favre-Réguillon, Alain

Feás, Xesús

Fedin, Matvey

Fedorova, Maria

Fedulov, Alexey V.

Fekete, Agnes

Felgueiras, Helena

Feng, Chia-Hsien

Ferens-Sieczkowska, Mirosława

Feresin, Rafaela G.

Ferlazzo, Nadia

Fernandes, Ana S.

Fernandes, Jolyn
Fernández Barbero, Gerardo

Fernandez, Bernadette $\mathrm{O}$.

Fernández, José A.

Fernandez, Mercedes

Fernández-García, M. Isabel

Fernández-Pérez, Leandro

Ferraboschi, Patrizia

Ferrante, Claudio

Ferrarese, Carlo

Ferrari, Silvia Martina

Ferreira, Paula M.

Ferreiro-González, Marta

Ferrer, Beatriz

Ferro, Domenico

Ferro, Riccardo

Ferroni, Lorenzo

Feveile Young, Jette

Fidalgo, Alexandra

Fifere, Adrian

Filep, János G.

Filgueira, Carly

Filip, Adriana

Filip, Lorena

Filip, Petr

Filipek, Anna

Filipovic, Milos R.

Fillmore, Paul

Fini, Mehdi A

Finley, John Weldon

Finno, Carrie

Fiorillo, Claudia

Firczuk, Malgorzata

Fiset, Sylvain

Fisher, Aron B.

Fiszer, Agnieszka

Flieger, Jolanta

Florek, Ewa

Florencio, Francisco J.

Flores-Bellver, Miguel

Florescu, Monica

Fogacci, Federica

Fogagnolo, Paolo

Fontanay, Stéphane

Fontanesi, Flavia

Fontanini, Debora

Fonte, Pedro

Fontés, Michel

Forero-Quintero, Linda S.

Foresti, Roberta

Forma, Ewa

Formanowicz, Dorota 
Formenti, Damiano

Fornai, Francesco

Forte, Elena

Forti, Luca

Fouda, Abdelrahman

Fox, Julie

Fraga, Cesar G.

Fraile, Lorenzo

François, Casas

Frank, Saša

Frankenberg-Dinkel, Nicole

Fratini, Filippo

Fredotović, Željana

Freire, M. Sonia

Frezza, Claudio

Frolov, Andrej

Frutos-Fernández, María José

Frydrychova, Radmila Capkova

Fthenakis, George

Fujii, Yoshiharu

Fujimori, Ko

Fujita, Masayuki

Fujiwara, Keigi

Fukudome, Akihito

Fulignati, Sara

Fulle, Stefania

Furdui, Cristina Maria

Fürnsinn, Clemens

Fusco, Roberta

Fuso, Andrea

Fuu-Jen, Tsai

Gabandé-Rodríguez, Enrique

Gabriel, Robert

Gac, Paweł

Gafencu, Anca

Gagaoua, Mohammed

Gago-Fuentes, Raquel

Gajewska, A.

Gajger, Ivana Tlak

Galanakis, Charis M.

Galaris, Dimitrios

Galatou, Eleftheria

Galbiati, Mariarita

Galea, Ian

Galic, Ante

Galindo-Villegas, Jorge

Galiniak, Sabina

Gall Troselj, Koraljka

Gáll, Zsolt

Galli, Francesco

Gallo, Monica
Galluccio, Michele

Gallyas, Ferenc

Galvez, Jorge

Gálvez, Julio

Gaman, Mihnea-Alexandru

Gamberi, Chiara

Gambini, Juan

Gamon, Luke Francis

Ganau, Mario

Ganesan, Kumar

Gantner, Magdalena

Garavaglia, Maria Lisa

Garbayo, Inés

García Gonzalo, Francesc

García, Concepción

García, Gabriela

García, José Joaquín

Garcia, Samuel

García-García, Rosa María

Garcia-Medina, José Javier

García-Otín, Ángel Luis

Garcia-Santamarina, Sarela

Gard, Paul

Gareth, Nye

Garrido-Fernández, António

Garten, Ryan

Garweg, Justus G.

Gasparrini, Massimiliano

Gates, Colin

Gautheron, Jérémie

Gawel, Kinga

Gaweł-Bęben, Katarzyna

Gawlik-Dziki, Urszula

Gazerani, Parisa

Gebicki, Jan

Gee, Heon Yung

Gęgotek, Agnieszka

Genovese, Claudia

Gentile, Carla

Gentile, Fabrizio

Geny, Bernard

Georgakilas, Alexandros

Georgiev, Georgi As.

Gerardi, Carmela

Gerasimenko, Oleg

Gerding, Heinrich

Gericke, Adrian

Germ, Mateja

Gerothanassis, Ioannis P.

Ghadiri, Maliheh

Ghag, Gaurav 
Ghiasi, Seyed Mojtaba

Ghidini, Michele

Giacomello, Emiliana

Giallauria, Francesco

Giannenas, Ilias

Gianotti, Andrea

Gieseg, Steven P.

Gil, Minchan

Giles, Gregory

Gilloteaux, Jacques

Gil-Martín, Emilio

Gil-Muñoz, Rocio

Giménez Bastida, Juan Antonio

Giordano, Antonio

Giordano, Anttonio

Giordano, Elena

Giordano, Maria

Giordano, Samantha

Giotta, Livia

Giovanna, Di Emidio

Giovannelli, Lisa

Giovannelli, Pia

Giovannoni, Roberto

Giovinazzo, Giovanna

Giri, Hemant

Girolami, Flavia

Giron-Gonzalez, Maria D.

Gismondi, Angelo

Giudetti, Anna

Giuffrè, Angelo Maria

Giuffrida, Daniele

Giulianini, Piero Giulio

Giuliano, Michela

Giunta, Salvatore

Giurisato, Emanuele

Giuseppina, Basini

Giustarini, Daniela

Giusti, Anna Maria

Giusti, Laura

Głąbska, Dominika

Gladysheva, Inna P.

Glibowski, Paweł

Gligor, Felicia

Gniazdowska-Piekarska, Agnieszka

Godfrey, Rinesh

Goffart, Steffi

Gohar, Eman

Goldman, Irwin L.

Gołębiowski, Tomasz

Golinelli, Marie-Pierre

Goljanek-Whysall, Kasia
Gomaraschi, Monica

Gómez Orte, Eva María

Gomez, Jesus

Gómez-Torres, Maria José

Gonçalves, Sandra

Gonkowski, Slawomir

Gonzalez, Antonio

González, Javier

Gonzalez, Michael J

González, Sonia

González-Álvarez, Julia

Gonzalez-Bulnes, Antonio

Gonzalez-Casado, Antonio

González-García, Jorge

González-Iglesias, Héctor

González-Manzano, Susana

González-Minero, Francisco José

González-Pérez, José A.

González-Redondo, Pedro

Goodwani, Sunil

Goraj, Weronika

Gorgoulis, Vassilis

Gori, Antonella

Gornas, Pawel

Gorniak, Patryk

Górnicka, Magdalena

Gorynski, Krzysztof

Goswami, Prabhat C.

Goto, Takaharu

Grabarczyk, Małgorzata

Graber, Tyson

Graczyk-Jarzynka, Agnieszka

Gradalski, Tomasz

Gradinaru, Robert

Gramignoli, Roberto

Granados-Principal, Sergio M.

Granica, Sebastian

Grant, George

Grasa, Laura

Grasso, Giuseppe

Graziella, Messina

Greenlief, C. Michael

Greetham, Darren

Gregić, Maja

Gregory, Stephen L.

Grider, Arthur

Grigoraš, Anca Giorgiana

Grigoriev, I. P.

Grigsby, Christopher L.

Grilli, Alfredo

Grimm, Marcus O. W. 
Grimm, Wolf-Dieter

Grossi, Giancarlo

Grosso, Giuseppe

Gruhlke, Martin C. H.

Grumati, Paolo

Gruzman, Arie-Lev

Grzegorczyk, Izabela

Grzelak-Błaszczyk, Katarzyna

Grzesiak, Jakub

Guerriero, Giulia

Guescini, Michele

Gugliandolo, Enrico

Guillén-Bejarano, Rafael

Guiné, Raquel

Gujski, Mariusz

Gulic, Tamara

Gulisano, Massimo

Gunn, Teresa

Guo, Bin

Guo, Chih-Hung

Guo, Fenghai

Gursoy, Ulvi

Gutierrez-Merino, Carlos

Gutsaeva, Diana

Guyonnet, Benoît

Guzmán, José Miguel

Gvozdjaková, Anna

Ha, Eunyoung

Haanes, Kristian

Habas, Khaled

Hafer, Carsten

Hailfinger, Stephan

Hajieva, Parvana

Halagarda, Michał

Haller, Steven T.

Hallmann, Ewelina

Hamaguchi, Masahide

Hamano, Tadanori

Hamilton, Chris

Hamilton, Shanna

Hammond, Billy R.

Hampton, Mark B.

Hanaka, Agnieszka

Hanania, Michel

Hancock, John

Hankinson, Oliver

Hano, Christophe

Hansen, Jason M.

Hansíková, Hana

Hanus-Fajerska, Ewa

Hardtke-Wolenski, Matthias
Hargreaves, Iain

Harrison, Sabine

Hartman, Mariusz

Harvey, Alexandra

Hasan, Raquibul

Hasegawa, Morifumi

Hasenstein, Karl H

Hashimoto, Yoshitaka

Hasse, Sybille

Hatae, Noriyuki

Hattinger, Claudia M.

Haybaeck, Johannes

Hazai, László

He, Quan

Hecel-Czaplicka, Aleksandra

Hegyi, Eszter

Heisinger, Stephan

Heitmar, Rebekka

Heller, Janosch

Henkel, Janin

Henry, Ryan A.

Her, Lu-Shiun

Herbet, Mariola

Hermesz, Edit

Hernan, Amanda

Hernández, Ángel

Hernández, Francisca

Herráez-Hernández, Rosa

Herrero, Enrique

Herrero, María Jesús

Hershfinkel, Michal

Hervás-Marin, David

Heydemann, Ahlke

Heyse, Alex

Higgins, Paul J.

Hildreth, Sherry

Hilhorst, Marc

Hinds, Terry D.

Hirata, Yoko

Hirota, Kiichi

Hirotaka, Kaneda

Hoffman, Jessica

Hoffmann, Michael Marcus

Hojs, Radovan

Hollenbach, Marcus

Holt, Roberta

Homa, Sheryl

Homma, Takujiro

Hong, Jiann-Ruey

Höög, Jan-Olov

Horstkorte, Rüdiger 
Hortigón-Vinagre, María

Hortobágyi, Tibor

Horvat, Daniela

Horváth, Eszter Mária

Horváth, Gabriella

Hošek, Jan

Hosohata, Keiko

Houng, Jer-Yiing

Hritcu, Lucian

Hsia, Shih-Min

Hsiao, George

Hsieh, Chang-Wei

Hsieh, Ming-Ju

Hsieh, Wen-Tsong

Hsin, Kun-Yi

Hsu, Tsai-Ching

Huang, Chun-Yung

Huang, Guan-Jhong

Huang, Huey-Chun

Huang, Hui-Chi

Huang, Shih-Yi

Huber, Otmar

Hubicka, Urszula

Huchzermeyer, Bernhard

Huertas, Jesús R.

Hung, Shao-Wen

Hung, Shih-Ya

Huppé-Gourgues, Frédéric

Hura, Tomasz

Husvéth, Ferenc

Hwang, In Koo

Hybertson, Brooks M.

Hytti, Maria

Ianora, Adrianna

Iapichino, Giovanni

Ibrahim, Salam

Ichii, Hirohito

Ichikawa, Hiroshi

Ientile, Riccardo

Igual, Marta

Iida, Kaoruko

Ilmarinen, Tanja

Im, Seung-Soon

Impellizzeri, Daniela

Imperatore, Concetta

Inagaki, Hidetoshi

Infascelli, Federico

Innchi, Lee

Inoue, Atsuko

Inoue, Hirofumi

Inoue, Kazuki
Ioannou, Irina

Ionescu, Elena Rodica

Ionut, Ioana A.

Irakli, Maria

Irato, Paola

Irena, Nalepa

Isemura, Mamoru

Ishikawa, Kiyotake

Ishitsuka, Yosuke

Islam, Mohammad Mirazul

Isola, Gaetano

Itabe, Hiroyuki

Ito, Fumiaki

Itoh, Ken

Itoh, Toshimasa

Iuliano, Rodolfo

Ivanova, Elena

Ives, Stephen

Iwai, Atsushi

Iwaoka, Michio

Iyer, Shilpa

Izdebska, Magdalena

Jaaro-Peled, Hanna

Jabłońska-Trypuć, Agata

Jaburek, Martin

Jacenik, Damian

Jacevic, Vesna

Jackson, William F.

Jadavji, Nafisa M.

Jaganjac, Morana

Jaime, Diego Franco

Jakobusic Brala, Cvijeta

Jakubczyk, Ewa

Jakubowski, Hieronim

Jala, Venkatakrishna Rao

Jamka, Małgorzata

Jamróz, Ewelina

Jamwal, Rohitash

Janda, Elzbieta

Janda, Katarzyna

Jang, Byoung Kuk

Janiszewska-Turak, Emilia

Jankauskienè, Julè

Janowiak, Blythe E.

Jantzie, Lauren L.

Jara Palacios, María José

Jarmuszkiewicz, Wiesława

Jarocka-Karpowicz, Iwona

Jarzebski, Maciej

Jastrzebska, Beata

Jaworowska, Agnieszka 
Jeandet, Philippe

Jedrejek, Dariusz

Jembrek, Maja Jazvinšćak

Jena, Prasant Kumar

Jeon, Sookyoung

Jerala, Nataša Kopitar

Jerebtsova, Marina

Jerkowić, Igor

Jeschke, Udo

Jeung, Eui-Bae

Ježek, Petr

Jiang, Lin-Hua

Jobe, Timothy

Joers, Valerie

Johnston, Carol

Jones, Meriel

Jong, Chianju

Joo, Nam-Seok

Jorge, Feito

José Tauler Riera, Pedro

Joshi, Pushpa Raj

Juhász, Tamás

Jukić Špika, Maja

Jumbo-Lucioni, Patricia

Jung, Young Sung

Jung, YoungSuk

Junichi, Kitanaka

Jurkowska, Halina

Juszczak, Lesław

Jyotsna, Mishra

Kabil, Omer

Kabir, Mohammad Faujul

Kaczmarek, Beata

Kadowaki, Daisuke

Kaether, Christoph

Kafarski, Pawel

Kafarski, Paweł

Kageyama, Hakuto

Kaja, Simon

Kajihara, Mikio

Kalayda, Ganna

Kale, Abhijit

Kalinin, Vladimir

Kaltsatou, Antonia

Kam, Antony

Kameshima, Satoshi

Kamieniczna, Marzena

Kamiński, Piotr

Kamolz, Lars-Peter

Kamzolova, Svetlana

Kanai, Masashi
Kanamori, Takao

Kanda, Naoko

Kandylis, Panagiotis

Kang, Dongchul

Kang, Junsu

Kang, Kyung Pyo

Kaňková, Kateřina

Kannan, Ram

Kant, Sashi

Kanugula, Anantha Koteswararao

Kao, Chai-Lin

Kappus, Rebecca

Kapur, Neeraj

Kapusta, Ireneusz

Kar, Adwitiya

Karcz, Dariusz

Kardassis, Dimitris

Kargl, Julia

Karikas, George-Albert

Karner, Courtney

Karwowska, Małgorzata

Kase, Satoru

Kashfi, Khosrow

Kashihara, Naoki

Kashiwakura, Ikuo

Kashtalap, Vasily

Kaškonienè, Vilma

Kataoka, Hiroyuki

Kato, Masaya

Kato, Takamitsu

Kato, Takamitsu A

Kato, Yasumasa

Katsoris, Panagiotis

Kauppinen, Anu

Kaur, Gurvinder

Kawakami, Susumu

Kawakita, Hidetaka

Kawamura, Takuji

Kawanami, Daiji

Kawata, Kazumi

Kawiak, Anna

Kayano, Shin-ichi

Kazimierczak, Renata

Keinänen, Markku

Kejík, Zdeněk

Kelleher, Shannon L.

Keller, Amy Celeste

Kello, Martin

Kensler, Thomas W.

Khan, Amjad

Khan, Mohsin 
Khan, Nabab

Khanna, Hemant

Khlebnikov, Andrei I.

Kicel, Agnieszka

Kicińska, Anna

Kieliszek, Marek

Kikowska, Małgorzata

Kikuchi, Hidehiko

Kikusato, Motoi

Kikuzaki, Hiroe

Kilanowicz, Anna

Kilari, Sreenivasulu

Kim, Bonglee

Kim, Donguk

Kim, Doyeon

Kim, Eun-Hee

Kim, Gi Jin

Kim, Hee Kee

Kim, Ho-Shik

Kim, Hyeung-Rak

Kim, Hyung-Sik

Kim, Jongmin

Kim, Ki Hyun

Kim, Kijin

Kim, Kil-Soo

Kim, Mi-hyun

Kim, Min-Hyun

Kim, Min-Soo

Kim, Nam Deuk

Kim, Seonghun

Kim, Seung-Hyun

Kim, Sun Yeou

KIM, Sung Joon

Kim, Sung-Hoon

Kim, Yong-Ick

Kim, Young-Mo

Kimura, Ken-ichi

Kind, Karen L.

Kinder, David

Kishikawa, Naoya

Kishimoto, Daiga

Kiss, Béla

Kiss, Rita

Klavins, Maris

Kleeff, Jörg

Kleier, Daniel A.

Klein, David C.

Klein, Erik

Kleinridders, André

Kleinstein, Sarah E.

Kleist, Thomas J.
Klotz, James

Kluczyk, Alicja

Kmiecik, Dominik

Knez, Damijan

Knezevic, Jelena

Ko, Gladys

KO, Kam Ming

Kobayashi, Satoru

Kobeissy, Firas

Kobus-Cisowska, Joanna

Koch, Wojciech

Koike, Haruki

Kojima, Hajime

Kojima-Yuasa, Akiko

Kokkola, Tarja

Kokoska, Ladislav

Kokoszyński, Dariusz

Kolasa-Wołosiuk, Agnieszka

Kolesar, Jill Marie

Kolesińka, Beata

Kolodziejczyk, Joanna

Kolosova, Nataliya

Komada, Munekazu

Komba, Shiro

Komis, George

Komoike, Yuta

Komsta, Łukasz

Konieczka, Paweł

Konopelski, Piotr

Konopka, Adam

Konopka, Iwona

Kontek, Bogdan

Kontogiorgis, Christos

Kontos, Christos K.

Kopacz, Aleksandra

Kopjar, Mirela

Koprowski, Piotr

Korać, Petra

Koryak, Yuri A.

Korybalska, Katarzyna

Kosmala, Monika

Kostecka-Gugala, Anna

Kosters, Astrid

Kostomitsopoulos, Nikolaos G.

Kostrzewa-Nowak, Dorota

Kostyn, Kamil

Kosuge, Yasuhiro

Kotani, Kazuhiko

Kouji, Harada

Koukounaras, Athanasios

Kouretas, Dimitrios 
Koutelidakis, Antonios E.

Kovac, Stjepana

Kowalczewski, Przemysław

Kowalczuk-Vasilev, Edyta

Kowalska, Jolanta

Kozakiewicz, Mariusz

Kozhevnikova, Oyuna

Koziorowska-Gilun, Magdalena

Kozlov, Andrey V.

Kozłowska, Mariola

Krajka-Kuźniak, Violetta

Krawczynski, Kamil

Krebs, Bernt

Krestinina, Olga

Krijt, Jan

Kristian, Tibor

Krokidis, Marios

Król, Ewelina

Krupa, Renata

$\mathrm{Ku}$, Kang-Mo

Kuan, Yu-Hsiang

Kuban-Jankowska, Alicja

Kubatka, Peter

Kubica, Paweł

Kubicova, Lenka

Kubinová, Renata

Kubinyi, Miklós

Kues, Wilfried A.

Kuhn, Hartmut

Kujawska, Małgorzata

Kukula-Koch, Wirginia

Kulbacka, Julita

Kulczyński, Bartosz

Kulminski, Alexander

Kumar, Akhilesh

Kumar, Vijay

Kumari, Asha

Kumari, Neelam

Kunz, Wolfram

Kuo, Cheng-Chin

Kuo, Ping-Chung

Kuo, Yao-Haur

Kurasaki, Masaaki

Kurganov, Boris

Kurihara, Yukio

Kurpisz, Maciej

Kursvietiene, Lolita

Kus, Piotr

Kushkevych, Ivan

Kuźnik, Nikodem

Kwak, Jong Hwan
Kwak, Seonyeong

Kwakowsky, Andrea

Kwiecien, Jacek

Kwon, Ki-Sun

Kypreos, Kyriakos E.

L. Pey, Angel

La Rosa, Piergiorgio

La Russa, Daniele

La Spina, Martina

La Vignera, Sandro

Labbé, Simon

Labbozzetta, Manuela

Labrou, Nikolaos

Labudda, Mateusz

Lacaille-Dubois, Marie-aleth

Lacham-Kaplan, Orly

Lachlan, Mitchell

Lachowicz, Sabina

Lacina, Lukas

Ladilov, Yury

Lagalwar, Sarita

Laganà, Antonio Simone

Lagouri, Vasiliki

Lahart, Ian

Lakk, Mónika

Lama, Adriano

LaMarca, Babette

Lamichhane, Santosh

Lamponi, Stefania

Lamy, Elsa

Landi, Marco

Landucci, Elisa

Langdon, Simon

Langton, Abigail K.

Lanner, Johanna T.

Lanza, Giuseppe

Lapteva, Maria

Larre, Isabel

Larson, Richard A

Lasoń, Władysław

Latek, Dorota

Latif, Sajid

Latino, Maria Elena

Latouche, Camille

Lattanzio, Rossano

Lauretani, Fulvio

Laurin, Jérôme

Laursen, Kristian Bruun

Lavandera, Jose Luis

Lavelli, Vera

Lavin, Martin F. 
Lavoie, Jean-Claude

Lawrenson, John

Lax, Pedro

Lazar, Zsofia

Le Clec'h, Winka

Le Guellec, Dominique

Le Romancer, Muriel

Le, Anh V

Lebaron, Richard

Lech, Krzysztof

Lechel, Andre

Ledgerwood, Elizabeth C.

Ledo, Ana

Lee, Ching-Kuo

Lee, Dae-Sung

Lee, Eun-Woo

Lee, Gihyun

Lee, Hong-Jin

Lee, Hui Jai

Lee, Jihyun

Lee, Jong-Jer

Lee, Junsoo

Lee, Junyeop

Lee, Kyung-Ah

Lee, Sang-Han

Lee, Sangho

Lee, Shin-Da

Lee, Sung Ki

Lee, Tzong-Shyuan

Lee, Tzu-Tai

Lee, Wing-Kee

Lee, Yong Sun

Lefevre, Sophie D.

Lehotský, Ján

Lei, Wei

Lei, Zhentian

Leicht, Christof

Leisz, Sandra

Leiva-Brondo, Miguel

Lenaz, Giorgio

Lendvai, Gábor

Leng, Tiandong

Lentini, Giovanni

Leon, Antonio

Leon, Francisco

León, Josefa

León, Rafael

Leone, Gemma

Leoni, Valerio

Leontopoulos, Stefanos

Lephart, Edwin D.
Les, Francisco

Lescure, Alain

Lesniak, Wieslawa

Levart, Alenka

Lever, Jeremie M.

Levy, Emile

Lewandowicz, Grazyna

Lewinska, Anna

Li, Chia-Jung

Li, Guohui

Li, Jine

Li, Lianbo

Li, Po-Hsien

$\mathrm{Li}$, Shiri

Li, Wei

Li, Xinbo

Liagre, Bertrand

Liao, Hung-Ju

Liao, Jyh-fei

Liao, Yung-Feng

Liby, Karen

Lim, Gareth E.

Lim, Kyung-Min

Lim, Tae-Gyu

Lima, Beatriz

Lima, Elisabete

Limauro, Danila

Lin, Chang-Shen

Lin, Chih-Li

Lin, Chun-Ping

Lin, Hai-Shu

Lin, Hsu Yang

Lin, Hui-Hsuan

Lin, Jer-An

Lin, Pei-Hui

Lin, Shih-Yi

Lin, Tung-Yi

Lin, Wei-Yong

Lin, Yuh-Yih

Lindsay, Angus

Lio, Do

Liobikas, Julius

Lionetti, Vincenzo

Lionetto, Maria Giulia

Liou, Ying-ming

Lipińska, Barbara

Litofsky, Norman Scott

Litvinova, Ekaterina A.

Liu, Chia-Chi

Liu, Chung-Jung

Liu, Shing-Hwa 
Liu, Yi-Chang

Liu, Yung-Chuan

Liu, Zijuan

Liverani, Elisabetta

Livrea, Maria Antonia

Lizard, Gérard

Lloyd, Matthew

Lo Muzio, Lorenzo

Lobanova, Ekaterina S.

Lobo, Gloria

Lobo, Joao

Loboda, Agnieszka

Lobysheva, Irina

Locato, Vitoria

Lodge, John K.

Lodovici, Maura

Lodyga-Chruscinska, Elżbieta

Lofrumento, Dario D.

Lognay, Georges

Lohan, Silke

Loizzo, Monica R.

Loizzo, Monica Rosa

Lombardo, Mauro

Lomonaco, Tommaso

Lonardo, Amedeo

Lončar, Mirela Baus

Lončarić, Ante

Longo, Pasquale

Longone, Patrizia

Lopez Domenech, Sandra

López Lluch, Guillermo

Lopez Olvera, Jorge Ramon

Lopez Rodilla, Jesus M.

López, Lucía Méndez

Lopez, Victor

Lopitz Otsoa, Fernando

Lorca, Ramon

Lordan, Ronan

Lorenc-Koci, Elżbieta

Lorenzo, Jose M.

$\mathrm{Lu}, \mathrm{Chi}-\mathrm{Yu}$

Lu, Mei-Chin

Lubrano, Valter

Luca, Giovanni

Lucarini, Massimo

Lucero, Diego

Łuczaj, Wojciech

Luddi, Alice

Ludwig, Bernd

Luís, Ângelo

Lukasiewicz, Marcin
Lukaski, Henry

Lukyanov, Pavel A.

Luna Maldonado, Aurelio

Lund, Elizabeth K.

Lung, Ming-Yu

Lunov, Oleg

Lupi, Saturnino Marco

Lupiáñez, José A.

Luzina, Olga

Lymberopoulos, Aristotelis

Lynch, Sean M.

M. Vorob'ev, Mikhail

Ma, Sihui

Maccallini, Cristina

Macchi, Beatrice

Machado Araújo, Maria Eduarda

Machin, Daniel R.

Maciejczyk, Mateusz

Mackrill, John

Madej, Dawid

Mademtzoglou, Despoina

Madigan, Michele

Madrid, Marisa

Madureira, Patricia

Maeng, Sung Ho

Maffei, Gianluca

Maffia, Michele

Magierowski, Marcin

Magni, Paolo

Maher, Pamela

Mahy, Jean-Pierre

Maietti, Annalisa

Mailloux, Ryan

Maio, Roberto Di

Maioli, Margherita

Maioli, Silvia

Maione, Francesco

Maiuolo, Jessica

Maixent, Jean Michel

Majima, Hideyuki J.

Majolino, Domenico

Makarova, Katerina

Makedou, Kali

Makishima, Makoto

Malapelle, Umberto

Malinska, Hana

Manam, Srikanth

Mancias, Joseph D.

Mancini, Antonio

Mancini, Ines

Mandal, Amritlal 
Mandatori, Domitilla

Mandrich, Luigi

Mandrioli, Roberto

Máñez, Salvador

Manfredini, Stefano

Manini, Paola

Manjunath, Manubolu

Mann, Francis

Mann, Giovanni

Manna, Caterina

Mannino, Giuseppe

Mantell, Casimiro

Mantzouridou, Fani

Marabini, Laura

Maranesi, Margherita

Marasco, Daniela

Marcal, Helder

Marchetti, Nicola

Marchini, Cristina

Marco-Contelles, José

Marek, Kieliszek

Marengo, Barbara

Maresca, Marc

Marfany, Gemma

Margaritelis, Nikos

Margină, Denisa

Mari, Michele

Maria Carmela, Bonaccorsi Di Patti

María Rosaura, Leis Trabazo

Marini, Federico

Marini, Herbert Ryan

Marino, Joseph

Marino, Tiziana

Mariot, Virginie

Mariscal-Arcas, Miguel

Markopoulos, Georgios S.

Marletta, Luisa

Marra, Alberto

Marracci, Silvia

Marrano, Nicola

Marsili, Stefania

Marsillach, Judit

Marsol, Alexis

Marszalek, Marta

Martelletti, Paolo

Martí, Sergio

Martikainen, Riikka

Martin, Eric

Martin, Maria

Martin-Bastida, Antonio

Martínez-Espinosa, Rosa María
Martínez-González, José

Martinez-Hervas, Sergio

Martinez-Martos, Jose Manuel

Martinez-Monteagudo, Sergio I.

Martinez-Pastor, Felipe

Martínez-Sánchez, Gregorio

Martín-Gil, Jesus

Martini, Daniela

Martins, Alice M.

Martins, Artur

Martins, Ian

Martins, Natália

Marty, Gary

Marullo, Salvatore

Maruyama, Kei

Masłyk, Maciej

Mast, Thomas

Mastinu, Andrea

Masurier, Nicolas

Mateeva, Nelly

Mateos Bernal, Rosa Maria

Materska, Małgorzata

Mateus, Maria L.

Mateus, Nuno

Mathew, Anna V.

Mathew, Mona

Matilla, Miguel

Matkowski, Adam

Matraszek-Gawron, Renata

Matsuda, Atsushi

Matsugo, Seiichi

Matsumoto, Yoshinori

Matsunami, Katsuyoshi

Matsuo, Muneaki

Matsuzaki, Shinya

Mattii, Letizia

Mattioli, Anna Vittoria

Mattoscio, Domenico

Matuszewska, Anna

Maugeri, Grazia

Maupin-Furlow, Julie A.

Mauriz, José L.

Mauro, Adolfo Gabriele

Maxwell, Jessie R.

Mayer-Proschel, Margot

Mayhan, William G.

Mayo, Juan Carlos

Mazur-Bialy, Agnieszka

McAllister, Matt

McDonald, Denise

McGill, Mitchell R. 
McMillan, JoEllyn M.

Mcmorrow, Tara

Md Badrul, Alam

Meaney, Steve

Mears, Jason

Meccariello, Rosaria

Medina, Reinhold J.

Mehariya, Sanjeet

Mehta, Sunali

Meléndez-Hevia, Enrique

Melini, Valentina

Meloni, Bruno

Mena, Salvador

Mencke, Rik

Méndez, Ana

Meneguzzo, Francesco

Menendez, Jose Carlos

Menghini, Luigi

Mennerich, Daniela

Merah, Othmane

Mercolini, Laura

Merecz-Sadowska, Anna

Merry, Troy

Mertas, Anna

Mesalam, Ahmed

Meslet-Cladiere, Laurence

Mesnage, Robin

Messner, Barbara

Messner, Donald

Metere, Alessio

Metzinger, Laurent

Meurman, Jukka

Meynier, Anne

Mézes, Miklós

Miastkowska, Małgorzata

Micale, Nicola

Micek, Agnieszka

Miceli, Natalizia

Michael, Adrian

Michaelis, Martin

Michalopoulos, Ioannis

Michalska, Anna

Michaud, Philippe

Michel, Piotr

Micheli, Laura

Michels, Alexander J.

Micillo, Raffaella

Micucci, Matteo

Mielcarek, Michal

Mieyal, John J.

Migliori, Carmela Anna
Miguel, Maria Da Graça Costa

Míguez, Jesús M.

Mikelis, Constantinos

Mikstacka, Renata

Mikulic-Petkovsek, Maja

Milano, Serena

Milardi, Danilo

Milković, Lidija

Millar, J. Cameron

Miloso, Mariarosaria

Mimura, Junsei

Min, Hyeyoung

Minami, Masaaki

Minato, Ken-ichiro

Minetti, Giampaolo

Miniero, Daniela Valeria

Minnelli, Cristina

Mioc, Marius

Miquel, Adrover

Miranda, Jonatan

Miranda, María

Mironeasa, Silvia

Mirza, Muhammad Usman

Misawa, Eriko

Mishima, Eikan

Mishra, Birendra

Mishra, Rosalin

Mitani, Takakazu

Mitchell, Scott G.

Mitliagka, Paraskevi

Miyamoto, Koji

Miyazawa, Shinichi

Miziak, Barbara

Mizobata, Tomohiro

Mladěnka, Přemysl

Mobbili, Giovanna

Moccia, Marcello

Modesti, Alessandra

Modica, Maria

Modun, Darko

Mogoșan, Cristina

Mohamed, Tamer

Mohanty, Joy G.

Moise, Alexander

Moldovan, Bianca

Moldoveanu, Serban C.

Molinaro, Antonio

Molins, Blanca

Mollace, Vincenzo

Mollen, Kevin P.

Mollica, Adriano 
Molmenti, Ernesto P.

Molnar, Arpad

Mołon, Mateusz

Momand, Jamil

Moncharmont, Bruno

Mondal, Kunal

Mondanelli, Giada

Montana, Giovanna

Montemiglio, Linda

Montenegro, Lucia

Montesano, Domenico

Montes-García, Verónica

Monticone, Silvia

Montiel-Duarte, Cristina

Montone, Carmela Maria

Monzón, Marta

Mookerjee, Shona

Moon, Jong-Seok

Moon, Minho

Moore, Roger A.

Moore, Tiffany A.

Morabito, Rossana

Morales Suárez-Varela, María M.

Morbidelli, Lucia

Morciano, Giampaolo

Moreno, Diego A.

Moreno, J.J.

Moreno, Maria João

Moreno, Sandra

Morgan-Bathke, Maria

Morikawa, Satoru

Morimura, Shigeru

Morrell, Jane M.

Morrow, John P.

Moschini, Roberta

Mosgöller, Wilhelm

Moshage, Han

Mosharov, Eugene V.

Moskalev, Alexey A.

Moskovitz, Jackob

Mosqueira, Matias

Mot, Augustin

Motterlini, Roberto

Mougios, Vassilis

Mousa, Shaaban A.

Mrakic-Sposta, Simona

Mravljak, Janez

Mshvildadze, Vakhtang

Muceniece, Ruta

Mucha, Joanna

Mücke, Ralph
Mueller, Wolf C.

Mugisho, Odunayo

Mu-Hsin, Chang

Mulas, Maurizio

Mulinacci, Nadia

Muller, Manfred James

Muller, Patricia A. J.

Müller-Schüssele, Stefanie

Muñiz, Pilar

Muntean, Danina

Munteanu, Florentina-Daniela

Mura, Maria Consuelo

Mura, Umberto

Murakami, Kohei

Murakami, Yasufumi

Murasami, Akira

Murase, Sachiko

Murata, Kazuya

Murawska-Ciałowicz, Eugenia

Murdaca, Giuseppe

Murdica, Valentina

Murphy, Ronan

Muscella, Antonella

Musco, Nadia

Mustachio, Lisa

Mustonen, Anne-Mari

Muszyńska, Ewa

Muszyński, Siemowit

Mutus, Bulent

Muzio, Giuliana

Muzolf-Panek, Malgorzata

Myasoedova, Veronika

Mysliveček, Jaromír

Nabika, Toru

Nabissi, Massimo

Nagahara, Noriyuki

Nagai, Takeharu

Nagata, Kosei

Nagy, Bálint

Nagy, Előd Ernő

Nagy, Istvan

Najera, Francisco

Nakajima, Ikuyo

Nakaki, Toshio

Nakamura, Motoki

Nakamura, Soichiro

Nakata, Masanori

Nakatani, Yoshihiko

Nakatsu, Kanji

Nalivaeva, Natalia

Namisaki, Tadashi 
Nandi, Shyam

Nannoni, Eleonora

Napolitano, Alessandra

Napolitano, Gaetana

Narayan, Mahesh

Narayanan, S. Priya

Nardecchia, Stefania

Nasim, Muhammad Jawad

Natarajan, Sivaraman

Nath, Arijit

Naumovski, Nenad

Navarro-Alarcon, Miguel

Navas, Placido

Naviglio, Daniele

Nawirska-Olszańska, Agnieszka

Neag, Maria Adriana

Neale, Joseph H.

Negishi, Takayuki

Negro, Carmine

Nemoto, Kiyomitsu

Nenadis, Niknen

Neuditschko, Markus

Neuman, Manuela

Neumann, Jake

$\mathrm{Ng}$, Hooi Hooi

Nicholas, Darrel

Nicoletti, Ferdinando

Niculescu, Loredan

Niederberger, Ellen

Nieto, Gema

Nieto, Marcelo J.

Nieva-Echevarría, Bárbara

Nikkanen, Lauri

Nikolakopoulou, Angeliki M.

Nioi, Claudia

Nishi, Kosuke

Nishi, Mayumi

Nishijima, Kazutoshi

Nishikawa, Yoshikazu

Nishimura, Koji

Nishimura, Naomichi

Nissen, Lorenzo

Niu, Tianhua

Niwano, Yoshimi

Njus, David

Noain, Daniela

Nobile, Stefano

Nocella, Cristina

Noda, Takeshi

Nofer, Jerzy-Roch

Noguchi, Constance Tom
Noguera Artiaga, Luis

Nogues, Isabel

Noh, Ji-yoon

Noren Hooten, Nicole

Noutsios, Georgios

Nouvian, Regis

Noviello, Carmine

Novikov, Arthur I.

Nowacka-Jechalke, Natalia

Nowak, Dorota

Nowak, Renata

Nowakowska-Zajdel, Ewa

Nowicka-Bauer, Karolina

Nowomiejska, Katarzyna

Nucera, Eleonora

Nunez, Oscar

Nunzio, Vicario

Nuzzo, Domenico

Nwaiwu, Ogueri

Nycz, Jacek E

Nylander, Karin

O'Keeffe-Ahern, Jonathan

O'Leary, Seán

O'Shaughnessy, Ryan

Obana, Akira

Oberemok, Volodymyr V.

Oberley, Rebecca

Obrador, Elena

Ochi, Shinichiro

Ochoa, Julio

Oczkowski, Michał

Oda, Shoji

Oddo, Elisabetta

Ogai, Kazuhiro

Ogi, Takayuki

Ogra, Yasumitsu

Oh, Jae-Wook

Ohnishi, Shiho

Oka, Shinichi

Okada, Hiroshi

Okajima, Hideaki

Oksenych, Valentyn

Okumura, Nobuaki

Olaru, Octavian Tudorel

Olech, Marta

Olennikov, Daniil

Oliveira, M. Conceição

Oliver, Peter L.

Omar, Syed Haris

Ondrias, Karol

Ong, Eng Shi 
Oniga, Ilioara

Oniszczuk, Anna

Opaliński, Łukasz

Oppedisano, Francesca

Oracz, Joanna

Orfila, Caroline

Orlacchio, Antonio

Orlando, Giustino

Oroian, Mircea Adrian

Orso, Evelyn

Orsolic, Nada

Ortega, Angel L.

Ortega, María Jesús

Ortega-Villaizan, Maria Del Mar

Orzelska-Górka, Jolanta

Osella, Alberto Rubén

Osredkar, Joško

Ostacolo, Carmine

Osuchowski, Marcin

Othman, Eman M.

Otręba, Michał

Otsuka, Yuzuru

Otulak-Kozieł, Katarzyna

Pacak, Christina

Pace, Paul

Pacifico, Severina

Pacini, Alessandra

Padilla-Benavides, Teresita

Pagano, Giovanni

Page, Melissa

Pagliarani, Alessandra

Pagonopoulou, Olga

Pak, Jhang Ho

Palacios-Santander, José María

Palade, Laurentiu Mihai

Palestini, Paola

Pallardó, Federico V.

Pallàs Lliberia, Mercè

Pallottini, Valentina

Palma, Miguel

Palmerini, Maria

Palmieri, Erika

Paltinean, Ramona

Palumbo, Giuseppe Alberto

Panagiotidis, Mihalis I.

Panaro, Maria Antonietta

Panfoli, Isabella

Pang, Cheng-Yoong

Pang, Myung-Geol

Pannella, Gianfranco

Pansarasa, Orietta
Pantaleo, Antonella

Pantea Stoian, Anca

Panzella, Lucia

Paola, Rosanna Di

Paoletti, Anna Maria

Paoli, Paolo

Paolino, Donatella

Papież, Monika

Papoutsis, Konstantinos

Pappa, Aglaia

Pappas, Christos

Parađiković, Nada

Parameshwaran, Kodeeswaran

Parenti, Astrid

Pariente Llanos, Jose Antonio

Park, Chan Young

Park, Han-A

Park, Joon Young

Park, Jun Hyoung

Park, Junsoo

Park, Kwang-hyun

Park, Ky Young

Park, Kyoung-Chan

Park, Kyung-soon

Park, Sang-Wook

Park, Shin-Hyung

Park, Songyoung

Park, Wansu

Parolini, Cinzia

Parry, Traci L.

Parsonage, Derek

Pârvu, Alina Elena

Pârvu, Marcel

Pasciu, Valeria

Pasman, Wilrike J.

Pasqualone, Antonella

Pastore, Analisa

Pastorelli, Grazia

Pateiro, Mirian

Patel, Alok

Pateras, Ioannis S.

Patruno, Antonia

Patruno, Cataldo

Pattappa, Girish

Patyra, Ewelina

Paudel, Bishal

Pauly, Diana

Pautz, Andrea

Paventi, Gianluca

Pavic, Valentina

Pavon-Djavid, Graciela 
Pawlak, Dariusz

Pawlas, Natalia

Pawliczak, Rafal

Pazdro, Robert

Pazzagli, Luigia

Peana, Massimiliano F.

Pecorelli, Alessandra

Peiretti, Pier Giorgio

Peixoto, Francisco

Pék, Zoltán

Pelle, Flavio Della

Pellegrini, Marika

Pellegrino, Daniela

Pellegrino, Roberto Maria

Peluso, Ilaria

Pence, Brandt

Penna, Claudia

Penning, Louis

Pepe, Salvatore

Perego, Paola

Pereira, David

Pereira-Leite, Catarina

Perera, Chamini J.

Perera, Conrad

Perestrelo, Rosa

Perestrelo, Rosa Maria De Sá

Pérez, Salvador

Pérez-Alvarez, Jose Angel

Pérez-Gálvez, Antonio

Pergolizzi, Simona

Perkins, Andrew

Perkovic, Ivana

Permyakov, Eugene

Pero, Raffaela

Perrotta, Cristiana

Persichilli, Silvia

Peskin, Alexander

Petcherski, Anton

Petit, Patrice X.

Petropoulos, Isabelle

Petropoulos, Spyridon A

Petrosino, Maria

Petrotos, Konstantinos

Petrucci, Rita

Petruczynik, Anna

Pezeshki, Adel

Pezzuto, Aldo

Philips, Neena

Piątkowska, Ewa

Piccinelli, Anna Lisa

Piccoli, Marco
Piekoszewski, Wojciech

Piemontese, Luca

Pierdomenico, Johnny Di

Pietrobelli, Angelo

Pietrzak, Wioleta

Pignatti, Patrizia

Pignitter, Marc

Pikija, Slaven

Pikuleva, Irina

Pilipenko, Vladimirs

Pilmane, Māra

Piluzza, Giannella

Pimienta, Rodney Lacret

Pina, Maria

Pinar, Anita

Pinchuk, Ilya

Pineda, Miguel

Piñeiro, Marta

Pinela, José

Pinheiro, Joaquina

Pini, Luigi Alberto

Pintea, Adela

Pintus, Eliana

Pintus, Gianfranco

Pinzaru, Iulia

Piomboni, Paola

Piotrowska, Katarzyna

Piotrowska, Małgorzata

Pirkmajer, Sergej

Pirman, Tatjana

Pirozzi, Claudio

Piscopo, Marina

Pittalà, Valeria

Piu, Saha

Pizent, Alica

Plastina, Pierluigi

Platania, Chiara

Plech, Tomasz

Ploner, Christian

Plumb, Jenny

Pluta, Ryszard

Pluth, Michael D.

Podgórska, Anna

Podsiadły, Radosław

Poeggeler, Burkhard

Pohl, Pawel

Pohl, Sebastian

Poiana, Marco

Poklar Ulrih, Nataša

Polacek, Norbert

Poli, Guiseppe 
Policar, Clotilde

Pöling, Jochen

Polonini, Hudson Caetano

Polyakov, Nikolay

Poma, Paola

Pompella, Alfonso

Pompili, Elena

Pompili, Simona

Pons, Toni

Poole, Leslie B.

Pop, Anca

Pop, Cristina

Popa, Daniela-Saveta

Popescu, Silvana

Porro, Chiara

Porteu, Françoise

Pósa, Anikó

Posadino, Anna Maria

Potaczek, Daniel P.

Potočnjak, Iva

Pourié, Grégory

Povey, Andrew

Pozsgai, Gabor

Prado, Armando Perez De

Prado-Cabrero, Alfonso

Pradotto, Luca Guglielmo

Prassl, Ruth

Prats, María Soledad

Pravenec, Michal

Prieto, Miguel Ángel

Prieto, Rafael Maria

Primack, William

Primavera, Rosita

Procopio, Jesús R.

Proestos, Charalampos

Proietti, Silvia

Przybył, Jarosław

Puglisi, Ivana

Pula, Giordano

Pulido, Olga M

Pulina, Giuseppe

Putilov, Arcady

Pyka, Alina

Pyka-Pająk, Alina

Qiu, Bin

Quaglino, Daniela

Quan, Taihao

Quartu, Marina

Quax, Paul

Quiles Morales, Jose L.

Quinlan, Leo R.
Quinn, Kylie

Quinto, Emiliano José

Quiros-Gonzalez, Isabel

R. El-Seedi, Hesham

Racay, Peter

Raccuia, Salvatore Antonino

Rachek, Lyudmila

Radak, Zsolt

Radko, Lidia

Radulescu, Cristiana

Raffaelli, Nadia

Rafińska, Katarzyna

Ragino, Yuliya I.

Ragusa, Andrea

Ragusa, Maria Antonietta

Rahimi, Farid

Rajala, Raju V.S.

Rajauria, Gaurav

Rajput, Vishnu D.

Ralph, Stephen John

Ramaiahgari, Sreenivasa C.

Ramalho-Santos, Joao

Ramaraj, Pandurangan

Ramírez, Carmen

Ramirez-Peña, Esmeralda

Ramos, Domingo

Ramos, Sonia

Rampon, Christine

Randall-Demllo, Sarron

Rankin, Gary

Ranucci, David

Ranzato, Elia

Rapeanu, Gabriela

Rasola, Andrea

Rattan, Suresh

Raugei, Giovanni

Ravanidis, Stylianos

Rawel, Harshadrai

Rayman, Margaret $\mathrm{P}$

Re, Lamberto

Rebollo-Hernanz, Miguel

Reeve, Vivienne

Regiec, Andrzej

Rekawiecki, Robert

Rembiałkowska, Ewa

Ren, Xiaoyuan

Renò, Filippo

Renshaw, Derek

Rescigno, Antonio

Restivo, Francesco M.

Restuccia, Donatella 
Retta, Saverio Francesco

Rety, Stephane

Rey, María-Dolores

Rhee, Jin-Kyu

Ribaudo, Giovanni

Ribeiro, Artur

Ribeiro, Daniela

Ribeiro-Barros, Ana I.

Ricci, Antonella

Ricci, Arianna

Riccò, Matteo

Richards, Dylan J.

Richter, Susan

Rieg, Timo

Riemer, Jan

Rijo, Patrícia

Rimbach, Gerald

Rinaldi, Carmen

Rinaldi, Teresa

Ringseis, Robert

Rinnerthaler, Mark

Rios-Covian, David

Riuzzi, Francesca

Rivas, Fatima

Rivera, Víctor M.

Rizvi, Syed A. A.

Rizzarelli, Enrico

Rizzi, Laura

Rizzo, Gianluca

Rizzolo, Anna

Roca, Jordi

Rocchetti, Gabriele

Rochani, Ankit

Roche, Enrique

Rochfort, Keith

Rodrigo, Sara Morales

Rodrigues, Francisca

Rodrigues, João

Rodrígues-Díez, Raquel

Rodrigues-Diez, Raul

Rodríguez Bernaldo De Quirós, Ana

Rodríguez, Soraya

Rodríguez, Víctor Manuel

Rodriguez-Lopez, Antonio D.

Rodriguez-Miguelez, Paula

Rodríguez-Solana, Raquel

Rodríguez-Yoldi, María Jesús

Rogalska, Justyna

Rogobete, Alexandru Florin

Roh, Miin

Roh, Sangho
Rohn, Sascha

Roje, Damir

Rokavec, Matjaz

Rolinec, Michal

Romanelli, Maria Grazia

Romani, Andrea

Romani, Patrizia

Romeo, Roberto

Romero, Alejandro

Romero, Francisco Javier

Roncarati, Alessandra

Rondeau, Philippe

Roosterman, Dirk

Rosa, Antonella

Rosado, Catarina

Rosca, Ana Maria

Rosi, Marzio

Rosic, Nedeljka

Ross, Ian

Ros-Santaella, Jose Luis

Rossi, Claudio

Rossi, Gianpaolo

Rossi, Jean-Christophe

Rossi, Miriam

Rosso, Chiara

Rostoker, Guy

Roth, Michael

Rothbauer, Mario

Rouault-Pierre, Kevin

Rouphael, Youssef

Rozalski, Marcin

Rua, Diego

Rudnitskaya, Ekaterina

Rudyk, Olena

Rudzińska, Magdalena

Ruf, Viktoria C.

Rui, Lixin

Ruiz Laza, Rocío

Ruiz Téllez, Trinidad

Ruiz, Mario

Rumbos, Christos

Rummey, Christian

Russo, Daniela

Russo, Marina

Russo, Mario Vincenzo

Rustad, Turid

Ruthstein, Sharon

Rutigliano, Mariacinzia

Ruzik, Lena

Rybczyńska-Tkaczyk, Kamila

Rybtsov, Stanislav 
Rymaszewska, Joanna

Ryu, Changseon

Ryu, Dongryeol

Rywaniak, Joanna

Rzemieniec, Joanna

Rzepecka-Stojko, Anna

Rzewuska, Magdalena

Saastamoinen, Markku

Sabatier, Jean-Marc

Sabatino, Laura

Saccà, Sergio Claudio

Sachadyn-Król, Monika

Sadowska-Krępa, Ewa

Sadowski, Martin

Saez, Carmen

Safirstein, Robert

Sahrawy, Mariam

Sahu, Ravi P

Saidu, Nathaniel Edward Bennett

Sainz, Rosa M

Saito, Satoshi

Saito, Yoshinobu

Saito, Yoshiro

Sak, Jarosław J.

Sakakibara, Hiroyuki

Sala, Gessica

Salas, José Blanco

Salas-Huetos, Albert

Saldanha, Sabita

Salemi, Michele

Salhab, Hassan

Salifoglou, Athanasios

Salinska, Elzbieta

Salmon, Adam

Salto-Gonzalez, Rafael

Salucci, Sara

Salvo, Andrea

Šamec, Dunja

Samhan-Arias, Alejandro

Samuels, Ivy

Sanak, Marek

Sánchez, Jesus Lozano

Sánchez-Bueno, Francisco

Sanchez-Quesada, José Luis

Sancho, M. Teresa

Sandbichler, Adolf

Sanfeliu, Coral

Sanjeewa, Kalu Kapuge Asanka

Sansbury, Brian E.

Sansone, Clementina

Santabarbara, J.
Santacroce, Luigi

Santanam, Nalini

Santi, Claudio

Santini, Antonello

Santoro, Massimo

Santos De Almeida, Tânia

Santos, Ana Cláudia

Santos, Andreia A.F.

Santos, Jerran

Santos, Renato

Santulli, Gaetano

Sanz, Ana

Sara, Franceschelli

Sarecka-Hujar, Beata

Saretzki, Gabriele

Sarsour, Ehab

Sartore, Giovanni

Sasaki, Yo

Sassoli, Chiara

Satake, Masayuki

Satish, Lakkakula

Sato, Takashi

Satoh, Masahiko

Satou, Tadaaki

Sattler, Wolfgang

Sattley, W. Matthew

Saurina, Javier

Savic, Ivan M.

Savion, Naphtali

Sawada, Koji

Sawada, Shojiro

Sawicki, Tomasz

Sazonova, Margarita A.

Scala, Valeria

Scalabrin, Elisa

Scalcon, Valeria

Scarafoni, Alessio

Scarlata, Eleonora

Schaefer, Michael K. E.

Schalinskie, Kevin

Schalkwijk, Casper

Schechter, Alan Neil

Schiavone, Achille

Schiavone, Marco

Schiavone, Stefania

Schiller, Jürgen

Schilling, Joel

Schinnerl, Johann

Schipper, Hyman M.

Schleicher, Erwin D.

Schlicker, Eberhard 
Schlüter, Klaus-Dieter

Schmidt, Ed

Schmitz, Matthias

Scholkmann, Felix

Schomburg, Lutz

Schöttker, Ben

Schuchardt, Mirjam

Schulze, Johannes

Schunke, Kathryn Jaques

Schwarzinger, Bettina

Ścibisz, Iwona

Scicchitano, Pietro

Scimone, Concetta

Scognamiglio, Monica

Scorziello, Antonella

Seca, Ana M. L

Sechi, Leonardo A.

Secondo, Agnese

See Hoe, Louise E.

Seeds, Michael C

Segatto, Marco

Segura, Inmaculada

Seiquer, Isabel

Sekara, Agnieszka

Sellars, Jonathan

SELLES, Benjamin

Seluanov, Andrei

Sendra, Esther

Senoner, Thomas

Senz Diez, María Teresa

Seo, Daisuke

Seo, Young-Kwon

Serefko, Anna

Sereikaite, Jolanta

Sergi, Consolato

Serio, Francesco

Serreli, Gabriele

Setyaningsih, Widiastuti

Severino, Paolo

Seydel, Karl

Sgherri, Cristina

Shackelford, Rodney E.

Shakeri, Majid

Sharma, Isha

Sharman, Edward H.

Shcherbik, Natalia V.

Sherlock, Laurie G.

Sherman, Philip

Shertzer, Howard G.

Shibata, Yasushi

Shieh, Tzong-Ming
Shienfeld, Yehuda

Shih, Jing-Wen

Shiina, Marisa

Shikama, Yosuke

Shikov, Alexander

Shim, Joon W.

Shimanouchi, Toshinori

Shimosawa, Tatsuo

Shin, Youngjae

Shmarakov, Igor

Shukitt-Hale, Barbara

Shukla, Kirtikar

Shults, Nataliia V.

Siatka, Tomáš

Siatkowski, Idzi

Šic Žlabur, Jana

Sicari, Vincenzo

Sidoti, Antonina

Sieber, Fritz

Sieniawska, Elwira

Signorile, Anna

Sigusch, Bernd W.

Sikalidis, Angelos

Sikazwe, Donald

Silaghi-Dumitrescu, Radu

Silswal, Neerupma

Silva Moura, Elisabete Ferreira

Silva, Amelia M

Silva, Branca

Silva, João Pedro

Silva, Paula

Silva, Pedro

Silvagno, Francesca

Silvestre, Armando J. D.

Silvestre, Samuel

Silvestri, Brigida

Silvestri, Cristian

Simal, Jesus

Simeonov, Vasil

Simes, Dina

Simitzis, Panagiotis

Simm, Andreas

Simó, Rafael

Šimoník, Ondřej

Simonini, Sara

Simón-Vázquez, Rosana

Sinanoglou, Vassilia

Singh, Abhishek

Singh, Anil

SINGH, JASHBIR

Singh, Pradeep 
Siniscalco, Dario

Siracusa, Rosalba

Siraki, Arno G.

Sjoholm, Ake

Skała, Ewa

Skendi, Adriana

Skirtach, Andre

Skoko, John J.

Skouta, Rachid

Skov, Vibe

Skovsted, Gry Freja

Skroza, Danijela

Skrypnik, Liubov N.

Skurikhin, Evgenii Germanovich

Slade, Dea

Slivka, Dustin Russel

Sliwinska, Agnieszka

Sliwinski, Tomasz

Sliwka, Hans-Richard

Słomińska-Wojewódzka, Monika

Slominski, Andrzej

Ślusarczyk, Sylwester

Smeriglio, Antonella

Smolen, Sylwester

Smolková, Katarina

Smuder, Ashley J.

Sneddon, Jennifer

Snowden, Timothy

Sobeh, Mansour

Sobiech, Przemysław

Sobierajska, Katarzyna

Sobocanec, CSandra

Sobota, Radoslaw M.

Socha, Robert

Solano, Francisco

Soler Valls, Ana Josefa

Soleti, Raffaella

Solingapuram Sai, Kiran Kumar

Somlyai, Gábor

Sone, Hideko

Song, Dae Kyu

Song, Hailong

Sonntag, Kai

Sorg, Olivier

Soriano, José Miguel

Soriano-Arroquia, Ana

Soriente, Alessandra

Sorokin, Vitaly

Sorrell, J. Michael

SORRENTINO, RAFFAELLA

Sosa Díaz, Teresa
Sousa, Maria João

Sousa, Mário

Soveral, Graça

Sowa, Ireneusz

Spallarossa, Andrea

Spampinato, Simona Federica

Spassov, Sashko

Spiers, Jereme

Spinelli, Francesco

Spingler, Bernhard

Spínola, Vítor

Spizzirri, U Gianfranco

Spletter, Maria

Squillacioti, Caterina

Šrámek, Jan

Srečec, Siniša

Srinivasan, Rahul

Srivastava, Sarika

Srivatsan, Malathi

Sroka, Zbigniew

Stacewicz-Sapuntzakis, Maria

Stadnyk, Andrew

Stagos, Dimitrios

Stahl, Wilhelm

Stanek, Agata

Stanila, Andreea

Stankova, Ivanka

Stanley, Jone A.

Stanley, Roger

Starčević, Antonio

Starowicz, Małgorzata

Starzak, Karolina

Stefanelli, Manuela

Stefania, Filosa

Stefano, Antonio Di

Stefanon, Bruno

Stefanowicz-Hajduk, Justyna

Stefanucci, Azzurra

Steinbrenner, Holger

Steiner, Joseph P.

Stellavato, Antonietta

Stepanic, Visnja

Steullet, Pascal

Stewart, Adele

Stilli, Donatella

Stipa, Pierluigi

Stiuso, Paola

Stixova, Lenka

Stobdan, Tsering

Stockmann, Christian

Stoian, Anca Mihaela Pantea 
Stojko, Jerzy

Stoleru, Vasile

Stone, William L.

Storchi, Paolo

Stornaiuolo, Mariano

Storsberg, Joachim

Stoyanova, Albena

Štrac, Dubravka Švob

Strappe, Padraig

Strati, Irini F.

Stratmann, Bernd

Stroffekova, Katarina

Strube, Jochen

Strungaru, Stefan-Adrian

Strychalski, Janusz

Strzemski, Maciej

Stuart, Jeffrey

Stubbs, Evan

Stushnoff, Cecil

Stutz, Bernardo

$\mathrm{Su}, \mathrm{Hua}$

Šubarić, Drago

Subramani Paranthaman, Balasubramani

Sud'ina, Galina F.

Suen, Jau-Ling

Sugawara, Akihiro

Suh, Hyung Joo

Suharoschi, Ramona

Sujak, Agnieszka

Sukseree, Supawadee

Suliburska, Joanna

Sulová, Zdenka

Suomela, Jukka-Pekka

Surdacki, Andrzej

Sureda, Antoni

Surguchov, Andrei

Suzuki, Katsuhiko

Suzuki, Norio

Suzuki, Shugo

Suzuki, Takafumi

Suzuki, Toshikazu

Svobodová, Jana

Svoronos, Paris D. N.

Świątek, Piotr

Świątkiewicz, Małgorzata

Świeca, Michał

Sykłowska-Baranek, Katarzyna

Symonds, Michael E.

Sytar, Oksana

Sytykiewicz, Hubert

Szakiel, Anna
Szaleniec, Maciej

Szászi, Katalin

Szatmari, Erzsebet Maria

Szepesi, Agnes

Szerszunowicz, Iwona

Szewczyk, Agnieszka

Szewczyk, Katarzyna

Szkaradkiewicz, Andrzej

Sznarkowska, Alicja

Szopa, Agnieszka

Szóstek-Mioduchowska, Anna

Sztanke, Małgorzata

Szulc-Dąbrowska, Lidia

Szumny, Antoni

Szutowicz, Andrzej

Szwajca, Anna

Szymandera-Buszka, Krystyna

Szymanski, Jedrzej

Tabuchi, Masashi

Tachibana, Yuya

Tada, Kohei

Tae-Jin, Kim

Tafuri, Simona

Tähtinen, Petri

Tai, Dar-Fu

Taiar, Redha

Taira, Junsei

Tajiri, Naoki

Takahashi, Hidenori

Takahashi, Hideyuki

Takahashi, Ken

Takano, Katsura

Takasawa, Shin

Takatani-Nakase, Tomoka

Takaya, Tomohide

Takayama, Fusako

Takegahara, Noriko

Takemori, Hiroshi

Takemura, Miho

Tamba, Bogdan Ionel

Tambuwala, Murtaza

Tanabe, Katsuyuki

Tanaka, Atsushi

Tanaka, Masaru

Tanase, Corneliu

Tang, Bor Luen

Tang, Soon Yew

Tanini, Damiano

Tanito, Masaki

Tantos, Ágnes

Taoka, Yousuke 
Tappia, Paramjit

Tarozzi, Andrea

TARRAGO, Lionel

Tasso, Bruno

Tatullo, Marco

Tava, Aldo

Tavazzi, Barbara

Tedesco, Idolo

Teiber, John

Tejero, Jesús

Telegina, Darya V.

Tena, Noelia

Teng, Ba-Bie

Tenore, Gian

Teodoro, João

Teragawa, Hiroki

Termini, John

Terrasson, Vincent

Terzaghi, William Bryan

Thackray, Alana

Thangavel, Chellappagounder

Thomas, Biju

Thomas, John

Thounaojam, Menaka Chanu

Thummuri, Dinesh

Tian, Shung Wu

Tiano, Luca

Tiboni, Gian Mario

Tilley, Douglas

Tiperciuc, Brindusa

Tirillini, Bruno

Titze, Jean

Toapanta, Franklin R.

Todorova, Dessislava

Toepel, Jörg

Tofalo, Rosanna

Toh, Ban-Hock

Tomaszewska, Ewa

Tonetti, Lorenzo

Tong, Junchao

Tonissen, Kathryn F

Tonni, Gabriele

Tonolo, Giancarlo

Torre, Maria Luisa

Torres Fuentes, Cristina

Torres Perez, María Dolores

Torres-Cuevas, Isabel

Tosti, Elisabetta

Toth, Peter

Tovar, Juscelino

Tower, John
Trabace, Luigia

Trabalzini, Lorenza

Traina, Giovanna

Tramonti, Angela

Travers, Jeffrey Bryant

Trcek, Janja

Trček, Janja

Trentini, Alessandro

Trevisan, Andrea

Tribulova, Narcis

Troedsson, Mats H.T.

Trofimov, Aleksei V.

TROMBETTA, MARIA

Troppmair, Jakob

Trucillo, Paolo

Trusca, Georgeta Violeta

Trzcińska, Monika

Tsai, James

Tsai, Jen-Chieh

Tsai, Kun-Lin

Tsai, Pei-Shiue

Tsai, Pi-Jen

Tsantarliotou, Maria P

Tsantili-Kakoulidou, Anna

Tscheliessing, Rupert

Tschernig, Thomas

Tseng, Chih-Hua

Tsopmo, Apollinaire

Tsoukalas, Dimitris

Tsubota, Akihito

Tsuchiya, Masahiko

Tufarelli, Vincenzo

Tundis, Rosa

Turano, Paola

Turrini, Federica

Tuttolomondo, Antonino

Tuuminen, Tamara

Tyan, Yu-Chang

Tzen, Jason T.C.

Tzeng, Chii-Reuy

Tzeng, Shiang-Jong

Uberti, Daniela

Uccelletti, Daniela

Uchida, Takeshi

Ufnal, Marcin

Ullah, Md Ashik

Umehara, Mikihisa

Urban, Milan

Urbanelli, Lorena

Urbaniak, Alicja

Urbanucci, Alfonso 
Urra, José Miguel

Ursoniu, Sorin

Usuki, Toyonobu

Uwai, Koji

Vacante, Marco

Václavíková, Radka

Vafiadaki, Elizabeth

Vaiman, Daniel

Vairetti, Mariapia

Vaisman, Boris

Valasi, Irene

Vale, Paulo

Valente, Nicola Alberto

Valenti, Maria

Valentine, Rudy J.

Valentová, Kateřina

Valenzuela, Juan Luis

Valles, Soraya L.

Valletta, Alessio

Vamanu, Emanuel

Van Der Laarse, Willem J.

Van Dyke, Michael

Van Gisbergen, Marike W.

Van Saen, Dorien

Ványolós, Attila

Váradi, Judit

Varikuti, Sanjay

Varillas, David

Vasicek, Ondrej

Vasileiadis, Ioannis

Vasin, Mikhail V.

Vassallo, Antonio

Vassallo, Neville

Vaudry, David

Vavvas, Demetrios G.

Vaz, Josiana A.

Vazquez, Guillerma

Veatch-Blohm, ME

Vega Alvarez, Jose Antonio

Vega, Jose Maria

Vejux, Anne

Velena, Astrida

Velentzas, Athanassios D.

Velkov, Zhivko

Vellecco, Valentina

Venckunas, Tomas

Venskutonis, Petras Rimantas

Veranic, Peter

Verardo, Vito

Vergara, Daniele

Vergara, M. Natalia
Verma, Anil K.

Vernetti, Lawrence A.

Verni, Michela

Verri, Tiziano

Versnel, Huib

Vestergaard, Mun'delanji C.

Vetrano, Ignazio Gaspare

Vetvicka, Vaclav

Vezzani, Bianca

Viapiana, Agnieszka

Vicario, Nunzio

Victor, Victor

Vieira, Helena L. A.

Vignes, Michel

Vignini, Arianna

Vijayakumar, Sarath

Vikulina, Anna

Vilela, Alice

Villa, Chiara

Villacampa, Mercedes

Villalta, Peter

Villani, Rehan M

Viña, José

Vinall, Ruth

Vinci, Cristina

Vinci, Maria Cristina

Vingolo, Enzo Maria

Vinson, Joe

Virag, Jitka A.I.

Virag, Laszlo

Virgili, Fabio

Virto, Mailo

Visconti, Roberta

Visentin, Michele

Vítámvás, Pavel

Vitetta, Luis

Vitiello, Peter

Vitorino, Rui Miguel Pinheiro

Vitturi, Dario

Viuda-Martos, Manuel

Vivarelli, Fabio

Vlassopoulos, Antonis

Voběrková, Stanislava

Vodnar, Dan C.

Volcho, Konstantin

Volkov, Vadim

Vomhof-DeKrey, Emilie

Von Ameln-Mayerhofer, Andreas

Von Knethen, Andreas

Von Lintig, Johannes

Vukojevic, Katarina 
Wada, Jun

Wada-Hiraike, Osamu

Wagner, Andreas

Wagner, Anika

Wagner, Kay-Dietrich

Wahli, Walter

Wan, Lei

Wang, Chao-Min

Wang, Chin-Kun

Wang, Hui-min

Wang, Jiongwei

Wang, Mong-Heng

Wang, Shin-Wei

Wang, Shu-Huei

Wang, Wen-Der

Warzecha, Zygmunt

Wassowicz, Wojciech

Watanabe, Mikiko

Weaver, Jolanta

Webb, R. Clinton

Wegwitz, Florian

Wei, Ming-Chi

Weiss, Siegfried

Weng, Ching Feng

Wenta, Tomasz

West, James D.

Weston-Green, Katrina

White, Roger

Whittaker, Peter

Wianowska, Dorota

Widemann, Emilie

Wieczorek, Edyta

Wiernicki, Ireneusz

Wiesner-Reinhold, Melanie

Wietstock, Philip

Wilczyński, Jacek

Wilde, Jerzy

Wilhelm Stahl, Wilhelm

Wilkins, Heather

Wilkinson, Jenny

Williams, Ryan

Willmore, William G.

Wińska, Katarzyna

Withers, Sarah

Witting, Paul K.

Wnuk, Agnieszka

Wnuk, Maciej

Wójciak, Karolina

Wójciak, Magdalena

Wójcik-Pszczoła, Katarzyna

Wojdylo, Aneta
Wojnowski, Wojciech

Wojtunik-Kulesza, Karolina Anna

Wojtyla, Łukasz

Woo, So-Youn

Wood, Jeff

Wood, Katherine

Woźniak, Gabriela

Woźniak, Łukasz

Wrześniok, Dorota

Wrzosek, Małgorzata

Wu, Chi-Rei

$\mathrm{Wu}$, Erxi

Wu, Gongwei

$\mathrm{Wu}, \mathrm{Shu}-\mathrm{Ju}$

$\mathrm{Wu}$, Wei-Li

$\mathrm{Xu}$, Maonian

Xuan, Tran Dang

Yadav, Pramod Kumar

Yadav, Vinita

Yager, Dorne

Yagishita, Yoko

Yakimova, Luidmila

Yamada, Sohsuke

Yamaguchi, Yusuke

Yamakoshi, Kimi

Yamakuchi, Munekazu

Yamamoto, Toshiharu

Yamamoto, Yusuke

Yamanaka, Keiichi

Yamasaki, Hideo

Yamashita, Atsushi

Yamauchi, Akira

Yang, Chang-Hao

Yang, Chuen-Mao

Yang, Chunzhang

Yang, Guangdong

Yang, Junhua

Yang, Shun-Fa

Yang, Wei-hsiung

Yano, Akira

Yao, Jian

Yasuda, Kazuki

Yee, Callista Stephanie

Yeh, Shu-Lan

Yen, Feng-Lin

Yen, Frances T.

Yeste, Marc

Yim, Soon-Ho

Yin, Jie

Ying, Mingyao

Yochum, Gregory S. 
Yoda, Satoshi

Yokoi, Fumiaki

Yoo, Yeong-Min

Yoon, Yisang

Yoshida, Go J.

Yoshino, Hironori

Yoshioka, Naoki

You, Hye Jin

You, Seungkwon

Youssefian, Leila

Ytrestøyl, Trine

$\mathrm{Yu}$, Chang Yeon

Yu, Kitaoka

Yu, Yung-Luen

Yuann, Jeu-Ming P.

Yudina, Lyubov

Yun, Hwi-Yeol

Yurchenko, Ekaterina

Yushenova, Irina

Zabad, Rana K.

Zabetakis, Ioannis

Zacchia, Miriam

Zaguła, Grzegorz

Zaid, Hilal

Zaitsev, Aleksey

Zakłos-Szyda, Malgorzata

Zakrzewski, Jerzy

Zalewska, Anna

Załuski, Daniel

Zammit Mangion, Marion

Zamyatnin, Andrey

Zanella, Isabella

Zanetic, Mirella

Zanón-Moreno, Vicente Calixto

Zapata Coll, Pedro Javier

Zapico, Sara Casado

Zapotoczny, Szczepan

Zaprutko, Lucjusz
Zarkovic, Neven

Zarnowski, Tomasz

Żarowska, Barbara

Zarrelli, Armando

Zarzycki, Pawel K.

Zawada, Katarzyna

Zawadzki, Jarosław

Żbikowska, Anna

Zdarta, Jakub

Zecca, Luigi

Zelina, Pavol

Zella, Davide

Zeng, Xiangkang

Zerbini, Gianpaolo

Zerva, Anastasia

Zhan, Yiqiang

Zhang, Congqiang

Zhao, Wei

Zhou, Aimin

Ziegler-Borowska, Marta

Zimmer-Bensch, Geraldine

Zingg, Jean-Marc

Zinnai, Angela

Ziogas, Vasileios

Ziolkowski, Wieslaw

Zitka, Ondrej

Zitko, Jan

Zitz, Ulrike

Ziyatdinova, Guzel

Złotek, Urszula

Zoidis, Evangelos

Zorov, Dmitry B

Zotti, Francesca

Zovko Končić, Marijana

Zucca, Paolo

Zuccari, Guendalina

Zugaza, José Luis

Żyżelewicz, Dorota 